\title{
Improving the Accuracy of the Adaptive Cross Approximation With a Convergence Criterion Based on Random Sampling
}

\author{
Alexander Heldring ${ }^{\circledR}$, Eduard Ubeda ${ }^{\circledR}$, Member, IEEE, and Juan M. Rius ${ }^{\circledR}$, Senior Member, IEEE
}

\begin{abstract}
The accuracy of the adaptive cross approximation (ACA) algorithm, a popular method for the compression of low-rank matrix blocks in method of moment computations, is sometimes seriously compromised by unpredictable errors in the convergence criterion. This article proposes an alternative criterion based on global sampling of the error in the elements of the ACA compressed matrix. The sampling error depends not only on the size of the sample but also on the population distribution of the error, which makes it difficult to control the error independently of the underlying problem. However, as argued and demonstrated in this article, the distribution of the error converges to the same unique probability distribution function for all low-rank matrices. Complementing the sampling criterion with a simple mechanism to detect this convergence, we arrive at a criterion that controls the error irrespective of the underlying problem. As a practical example, the RCS of a moderate size metallic ogive is computed to illustrate the merits of the proposed criterion. The proposed algorithm may also be useful in other methods that approximate low-rank matrices by interpolation of a reduced set of its elements.
\end{abstract}

Index Terms-Adaptive cross approximation (ACA), computational electromagnetics, fast solvers, method of moments.

\section{INTRODUCTION}

$\mathbf{T}$ HE adaptive cross approximation (ACA) algorithm [1] is a rank revealing matrix decomposition that has received widespread attention in the computational electromagnetics community since its introduction to the field in 2005 [2]. This success is due to its capacity to generate, efficiently and reliably, a blockwise compressed representation of the method of moments impedance matrix. The core of the algorithm is a simple and elegant pivoting strategy that optimizes what is essentially an ordinary LU decomposition by picking, with every iteration, those rows and columns of the original block that are expected to contribute most to the convergence of

Manuscript received October 21, 2019; revised May 11, 2020; accepted June 5, 2020. This work was supported in part by FEDER, in part by the Spanish Plan Estatal de Investigación Científica y Técnica y de Innovación", under Grant TEC2016-78028-C3-1-P, Grant TEC2017-83343-C4-2-R, and Grant TEC2017-84817-C2-2-R, in part by the Unidad de Excelencia Maria de Maeztu MDM-2016-0600, which is financed by the Agencia Estatal de Investigación, Spain, and in part by the Catalan Research Group 2017 under Grant SGR219. (Corresponding author: Alexander Heldring.)

The authors are with the AntennaLab, Department of Signal Processing and Telecommunications, Universitat Politecnica de Catalunya, 08034 Barcelona, Spain (e-mail: heldring@tsc.upc.edu).

Color versions of one or more of the figures in this article are available online at http://ieeexplore.ieee.org.

Digital Object Identifier 10.1109/TAP.2020.3010857 the algorithm. It also allows formulating a convergence criterion that estimates the residual error of the current decomposition at every iteration and thus decides when to declare convergence and stop iterating further. The convergence criterion makes an estimation of the difference between the decomposition and the original matrix block. Of course, since one of the strengths of the ACA is precisely that only those few rows and columns that actually contribute to the decomposition are computed, a large part of the original block remains unknown, leading to an inherent uncertainty in the convergence criterion. As a consequence, convergence is sometimes declared when the error is more than an order of magnitude above the chosen threshold. This issue has been pointed out in the literature before [3], [4], but it has never been adequately addressed. Indeed, one may argue that it is an inevitable consequence of using an approximation and must simply be accepted as such. Furthermore, the error in any given matrix subblock will be only qualitatively related to the error in the solution of the method of moments linear system, so the choice of convergence threshold is somewhat arbitrary anyway. Nevertheless, we believe that an alternative formulation that yields a more reliable minimum accuracy would be a welcome improvement, provided that it does not compromise the overall efficiency of the ACA.

In this article, we propose an alternative convergence criterion that satisfies these requirements. The new criterion is based on random sampling of the error. In order to ensure that the estimate from the sample is close to the true value, we independently monitor the population distribution. Crucially, we show that after sufficient iterations, this distribution must converge to a unique probability density function. If necessary, we continue iterating until this state is reached.

In this article, the proposed method is incorporated in the ACA algorithm, but it may be useful for estimating the residual error in any algorithm that approximates low-rank matrices by interpolation of a reduced set of its elements. Such algorithms are used in a wide field of scientific applications. Some examples are randomized QR decomposition for parameter estimation [5], the CUR-decomposition for data analysis [6], or the mosaic-skeleton method [7] for solving integral equations.

This article is organized as follows. In Section II, a review of the conventional ACA convergence criterion is given. In Section III, the new criterion is derived. In Section IV, 
the statistical distribution of the error matrix elements is analyzed. In Section V, the new criterion is complemented with information from the statistical distribution. Section VI presents a practical example, and Section VII presents some conclusions.

\section{Conventional ACA Convergence CRiterion}

In ACA, the matrix to be compressed, $A$ of size $m \times n$, is approximated as $A \approx U_{k} V_{k}^{T}$, where $U_{k}$ and $V_{k}$ are, respectively, of size $m \times k$ and $n \times k$, with $k \ll m, k \ll n$, hence the compression. Matrices $U_{k}$ and $V_{k}$ are built iteratively by adding a column $u_{k}, v_{k}$ at each iteration. These are computed from a selected row and column of the block $A$ to be compressed, of indices $I_{k}$ and $J_{k}$, respectively, called the pivots, which are the worst-represented by the current decomposition (have the largest error). When $u_{k}$ and $v_{k}$ are incorporated in the decomposition $U_{k} V_{k}^{T} \approx A$, the corresponding row $I_{k}$ and column $J_{k}$ of the error matrix $E_{k}=U_{k} V_{k}^{T}-A$ are reduced to zero.

The convergence criterion proposed in the original ACA paper [1] works as follows. Assuming the original block to be low-rank, at some stage, the decomposition will cover the full row and column space of $A$ and the Frobenius norm of the error matrix, $\left\|E_{k}\right\|_{F}$, will decrease exponentially with every subsequent iteration. The error matrix can then be estimated from the currently selected column $u_{k}$ and row $v_{k}$ as $E_{k}^{(e s t i m)}=u_{k} v_{k}^{T}$. Since the variable of interest is the relative error, the absolute error $\left\|E_{k}\right\|_{F}$ must be divided by the Frobenius norm of $A$ itself, which is approximated by the Frobenius norm of $U_{k} V_{k}^{T}$. In summary, the convergence criterion compares the estimated relative error at each step $k$

$$
\epsilon_{k}^{\prime}=\frac{\left\|u_{k} v_{k}^{T}\right\|_{F}}{\left\|U_{k} V_{k}^{T}\right\|_{F}}
$$

with a chosen threshold $\tau$ rather than the true relative error

$$
\epsilon_{k}=\frac{\left\|E_{k}\right\|_{F}}{\|A\|_{F}}
$$

which would be much too computationally expensive.

Since the selection of new rows and columns relies on the current state of the decomposition, it does not guide the choice of the first row pivot at the start of the iterations, $I_{1}$. The original ACA paper [1] does not specify where to start, while [2] presents the algorithm with the row of index one as a starting point [see the algorithm described in Section V (line 3)]. The open ACA literature provides no alternative suggestions. It is clear that this introduces an element of randomness in the ACA algorithm, albeit somewhat obfuscated since the random choice is made only once, at the moment, the algorithm is implemented.

In order to illustrate the effect of this choice on the accuracy of the ACA, we show in Fig. 1 the histogram of the true relative error of an ACA decomposition for all possible choices of the starting row, after convergence to a threshold value of $\tau=10^{-3}$. The underlying model consists of two square metal plates positioned as shown in the inset. The block $A$ to be compressed is the mutual impedance matrix of the two plates, formulated with the electric field integral equation

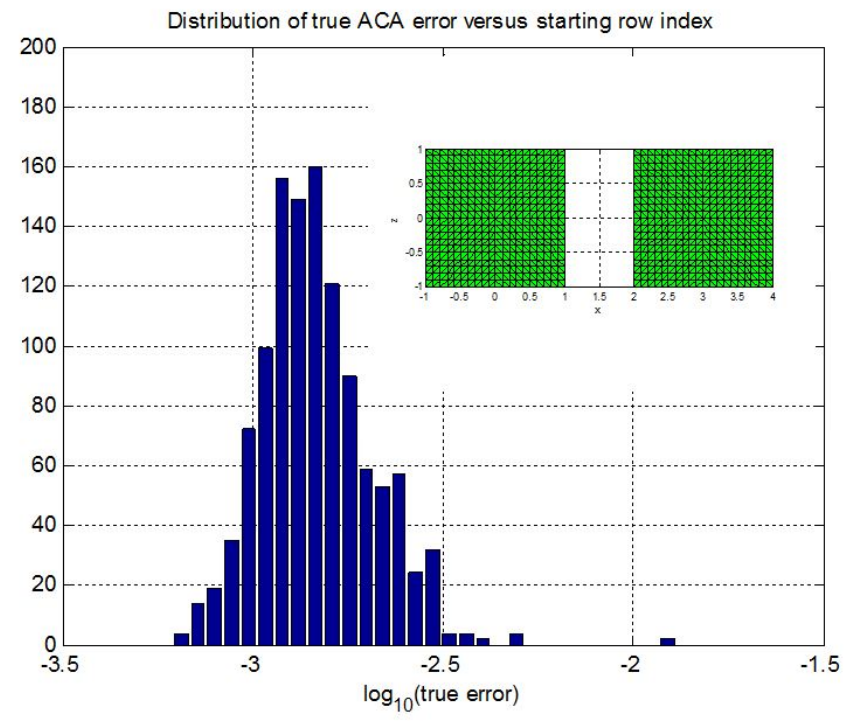

Fig. 1. Histogram of the true relative error of the ACA compression of the mutual impedance of two square plates, for all 1160 initial row indices, with an ACA accuracy threshold of $10^{-3}$.

and discretized using RWG basis functions [8]. The plate dimensions are $2 \lambda \times 2 \lambda$, the distance between plates is $1 \lambda$, and the triangularization is regular, with 1160 edges of only two different lengths, $0.1 \lambda$ and $0.14 \lambda$ on each plate.

One observes that the average true relative error is around $10^{-2.8}$, only slightly larger than the threshold, but there is one case with a true relative error of $10^{-1.9}$, more than ten times the threshold. Such cases are not always present, and their occurrence as a function of wavelength, geometry, or initial index choice is completely unpredictable. The example mentioned earlier was found after a few tryouts with square plates in different positions, by no means an exhaustive search for the worst case. We must assume that the true relative error can be far worse still.

As mentioned in Section I, the error in a given impedance matrix subblock does not linearly translate to the error in the full system solution. Since only blocks representing nontouching groups of basis functions are compressed, the overall solution will not suffer much in the case of well-conditioned, diagonally dominant impedance matrices. When, on the other hand, the impedance matrix is poorly conditioned, the error in off-diagonal blocks may get amplified during the system solution.

As an example, we use ACA to solve the problem of a $1 \mathrm{~m}$ length by $10-\mathrm{cm}$-diameter open metal cylinder, discretized with 11980 RWG basis functions, subdivided into ten groups with 1198 basis functions each, as shown in Fig. 2. The cylinder is illuminated by a plane wave, propagating perpendicular to the cylinder axis, at $150 \mathrm{MHz}$. The matrix blocks representing the interaction between nontouching groups are ACA-compressed with a threshold of $10^{-3}$. The system is solved using GMRES [9] with a threshold of $10^{-6}$, a total of 1198 times, with the initial row index for all the ACA compressions incremented by one with each solution. Fig. 2 shows the relative difference with the exact MoM solution in the 


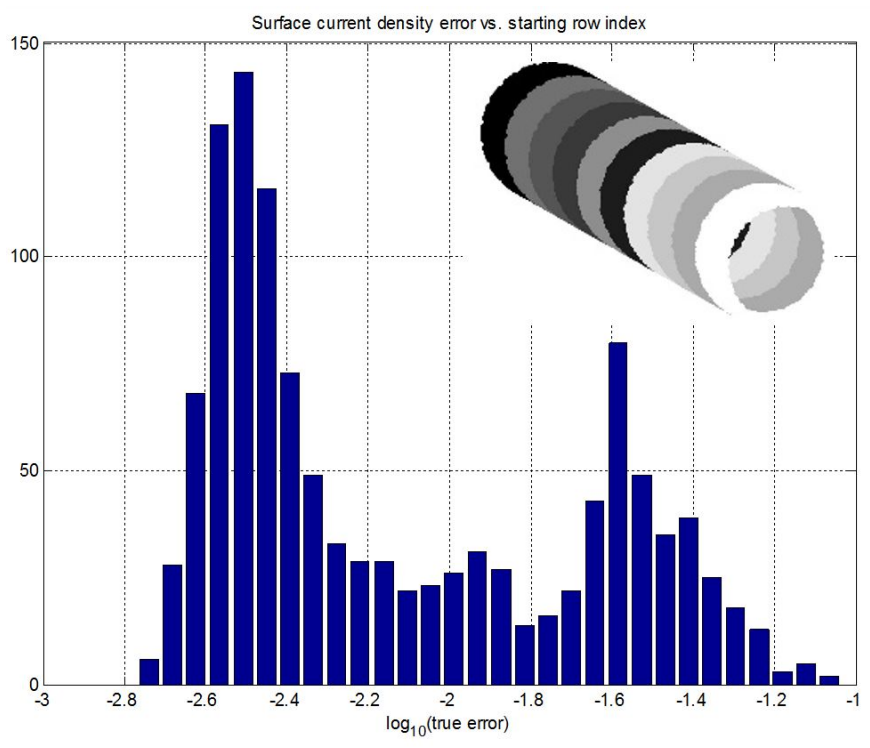

Fig. 2. Histogram of the relative error in the surface current density on a metal cylinder, computed with MoM-ACA, as a function of the ACA initial row index. Inset: geometrical decomposition of cylinder for ACA subblock compression.

norm of the surface current vector for each run. One observes that it varies by almost two orders of magnitude.

\section{New ACA CONVERGENCE CRITERION}

After trying in vain to establish a relation between the choice of the initial row index and the true relative error at convergence, we decided instead to make the statistical nature of the convergence criterion explicit, in a controlled manner. We observe that the square of the Frobenius norm of a matrix is proportional to the mean value of the squared norms of the matrix elements. This means that the sample mean of a simple random sample of size $M \geq 1$ of the squared element norms is an unbiased estimator for the Frobenius norm. The issue is then how to take a sample from $E_{k}$, which changes with every iteration and is never explicitly computed.

Consider the ACA decomposition of an $m \times n$ matrix block $A$ with convergence threshold $\tau$. Before starting the ACA iterations, we generate a vector $\boldsymbol{r}$ of random row indices $r_{i}, i=1 \ldots M$ and a vector $\boldsymbol{c}$ of random column indices $c_{i}, i=1 \ldots M$ (with replacement). Then, we compute the vector $\boldsymbol{a}$ of $M$ matrix elements corresponding to each pair of indices $A\left(r_{i}, c_{i}\right)$. Now, after each ACA iteration, we are interested in the vector $\boldsymbol{e}_{\boldsymbol{k}}$ defined by the elements indexed $(\boldsymbol{r}, \boldsymbol{c})$, of the error matrix $E_{k}$. Initially, $U$ and $V$ are void, so $\boldsymbol{e}_{0}=\boldsymbol{a}$. Subsequently, each ACA iteration yields two vectors $\boldsymbol{u}_{\boldsymbol{k}}$ and $\boldsymbol{v}_{\boldsymbol{k}}$ to be incorporated in the matrices $U_{k-1}$ and $V_{k-1}$ of the previous approximation. It is easily verified that $\boldsymbol{e}_{\boldsymbol{k}}$ can be recursively computed, without any additional matrix element evaluations after the initial calculation of $\boldsymbol{a}$, with

$$
\boldsymbol{e}_{\boldsymbol{k}}(i)=\boldsymbol{e}_{\boldsymbol{k}-\mathbf{1}}(i)-\boldsymbol{u}_{\boldsymbol{k}}\left(r_{i}\right) \boldsymbol{v}_{\boldsymbol{k}}\left(c_{i}\right), \quad i=1 \ldots M .
$$

The mean $v_{k}$ of the squared moduli of the elements of $\boldsymbol{e}_{\boldsymbol{k}}$ is a random variable with an unknown distribution, whose

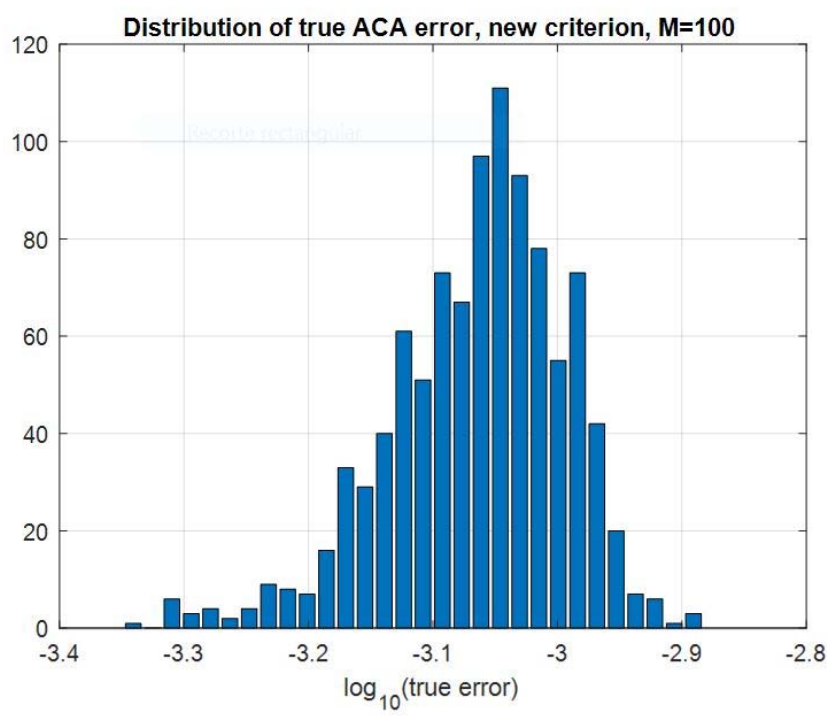

Fig. 3. Histogram of the true relative error of the ACA compression for the same problem as in Fig. 1 with the proposed new criterion, 1.000 runs with sample size $M=100$.

expectation $\mu_{k}$ equals the mean value for the full population matrix $E_{k}$, which is proportional to the squared Frobenius norm: $\left\|E_{k}\right\|_{F}^{2}=n m \mu_{k}$.

The statistical uncertainty in using $v_{k}$ as an estimator for $\mu_{k}$ depends on the sample size $M$ and the distribution of the elements of $E_{k}$. Unfortunately, this distribution changes drastically and unpredictably with every iteration, so we cannot characterize it without compromising the overall efficiency of the algorithm. Besides, even if we could, it would be impractical to adapt the sample size with every iteration. All we know is that the choice of $M$ will be a compromise between the uncertainty and the efficiency. We, therefore, choose a value that is sure to have very little impact on the overall efficiency, say $M=100$, and see what happens.

First, we apply it to the problem of Fig. 1. The histogram of 1.000 executions of this experiment is shown in Fig. 3. The observed negative skew and the fact that the mean value is somewhat below $10^{-3}$ are due to an overshoot effect. The estimated relative error changes in discrete steps; therefore, at convergence, it is never exactly $10^{-3}$, and instead, it is randomly distributed below that value. This introduces an inevitable spread in the estimated relative error. If, for instance, we increased the sample size to $M=1.000$, we would reduce the right tail of Fig. 3, but the spread due to the overshoot would still be there.

We have repeated the experiment with the cylinder of Fig. 2 with the new criterion and $M=100$, and again, the result, shown in Fig. 4, represents a very important improvement over the conventional approach.

The two experiments mentioned earlier involve matrix blocks with relatively homogeneous squared norms of the elements of the matrix blocks. Since the error matrix $E_{k}$ is built up of linear combinations of the rows and columns of the blocks, it seems reasonable to assume that this homogeneity will carry over in $E_{k}$. The spread around the mean, 


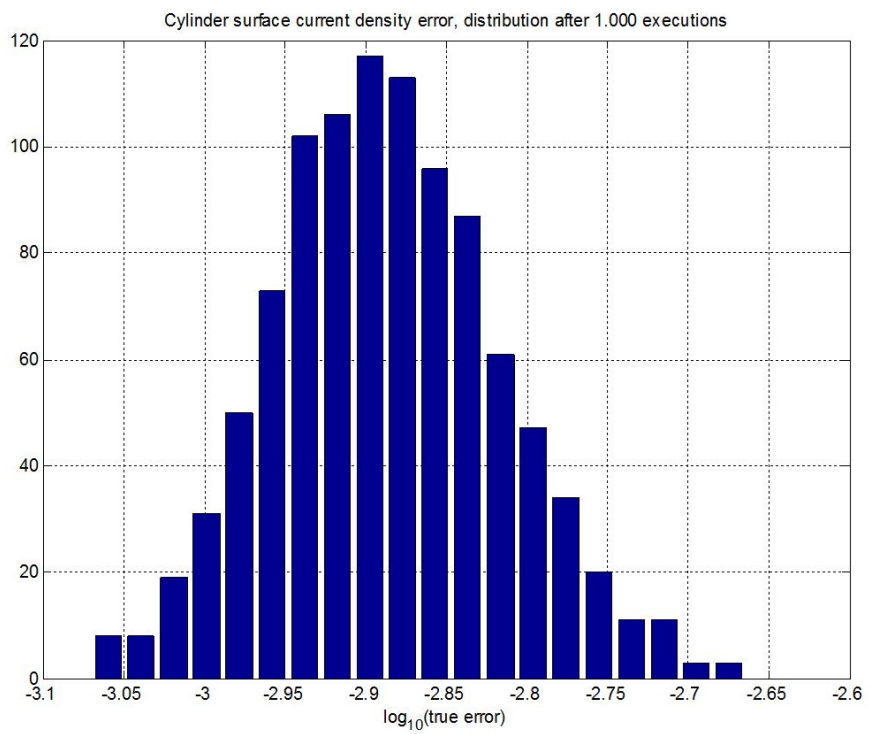

Fig. 4. Histogram of the relative error in the surface current density on the metal cylinder of Fig. 2 with the proposed new criterion, 1.000 runs with sample size $M=100$.

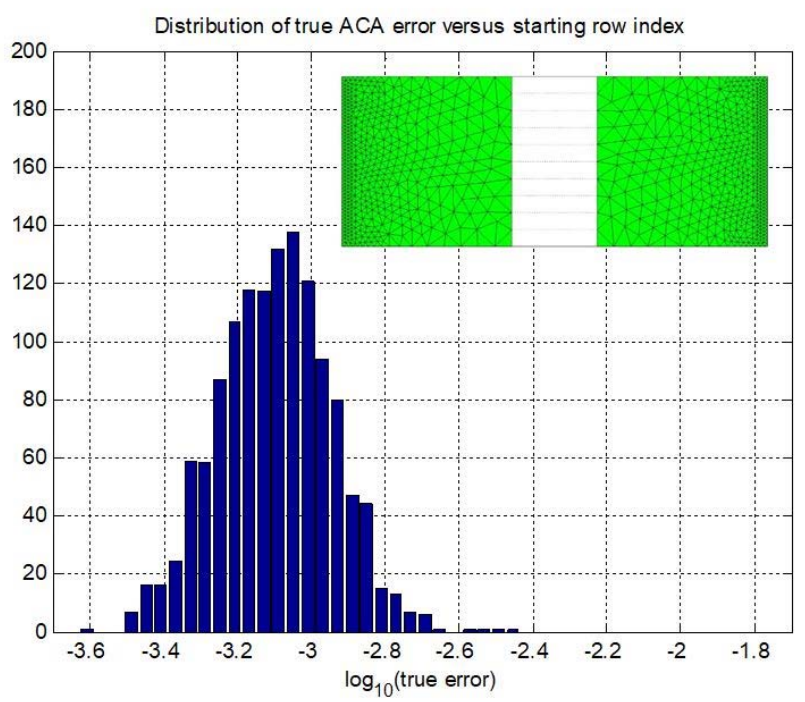

Fig. 5. Histogram of the true relative error of the ACA compression of the mutual impedance of two square plates, irregularly meshed, for all 1312 initial row indices. ACA threshold $\tau=10^{-3}$

or dispersion, of the elements of $E_{k}$ can, therefore, be expected to be relatively mild, which explains why good results are obtained with a sample size as small as $M=100$. This is not always the case. The magnitude of the elements of $A$ depends on features, such as the mutual distance between basis functions, their relative orientation, and the relative sizes of the corresponding triangles.

In order to investigate the behavior under more challenging conditions, we have replaced the mesh of the plates of Fig. 1 with the very irregular mesh of the inset in Fig. 5. The same figure shows the distribution of the true relative error using the conventional ACA criterion, for all initial row indices. We observe that the extreme outliers are absent this time;

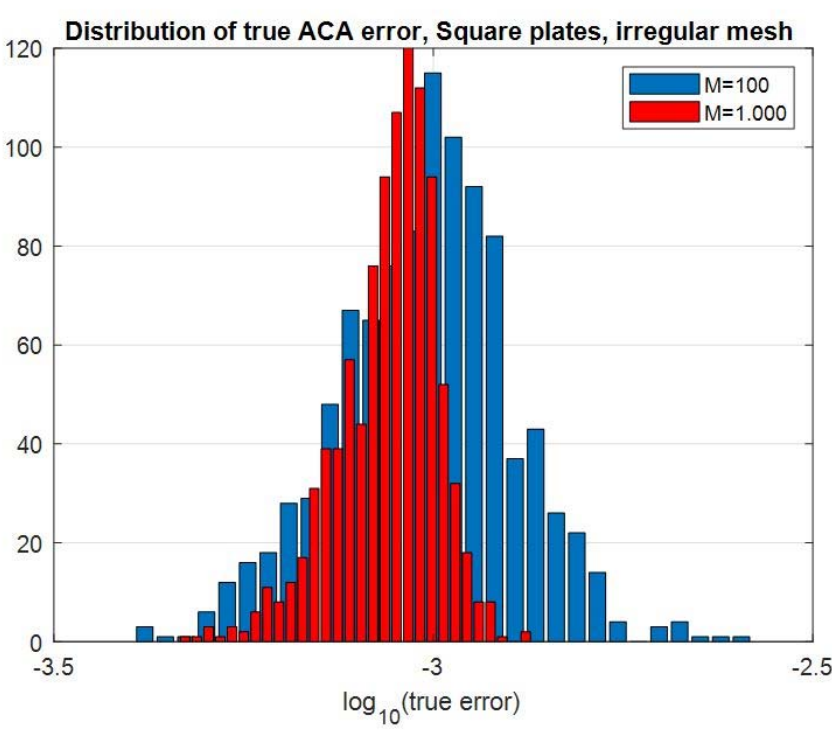

Fig. 6. Histogram of the true relative error of the ACA compression for the same problem as in Fig. 5, with the proposed new criterion, 1.000 runs.

as mentioned earlier, they do not always occur and their cause remains unexplained.

In Fig. 6, the distribution of the true relative error after 1.000 runs with the new criterion is shown. As expected, with sample size $M=100$, the spread is far higher than with the regular grid of Fig. 1, although it is still lower than with the conventional ACA criterion. Also shown is the distribution when the sample size is increased to $M=1.000$. This last distribution is comparable to the one for the regular grid with $M=100$. Unfortunately, we cannot reliably estimate the correct choice of $\mathrm{M}$ in advance. The only option in order to arrive at a truly problem-independent criterion is to try and find some universal characteristics of the distribution of the elements of $E_{k}$. This will be the subject of Section IV.

\section{ON THE Distribution OF THE ElEMENTS OF THE ACA ERROR MATRIX}

After iteration $k$ of the ACA algorithm, we have for the error matrix $E_{k}$

$$
E_{k}=A-\sum_{i=1}^{k} \mathbf{u}_{i} \mathbf{v}_{i}^{T}
$$

where $\mathbf{u}_{i}$ and $\mathbf{v}_{i}$ are the selected rows and columns of $A$, orthogonalized with respect to all the previous $\mathbf{u}_{i}$ and $\mathbf{v}_{i}$. Since these two are constructed so as to completely cancel the existing error for the entire corresponding row and column, they are in fact, up to a minus sign, equal to this error. Hence, at every step, we have access to one row and one column of $E_{k}$. The conventional convergence criterion is based on the assertion that after some step $k=K$, the full rank of $A$ is covered, and beyond this step, the true relative error drops exponentially with $k$, which in turn implies that every subsequent adaptation of $E_{k}$, equal to $\mathbf{u}_{k} \mathbf{v}_{k}^{T}$, must be at least of the order of magnitude of $E_{k}$ itself. Up until 
$k=K$, little can be said about the distribution of $E_{k}$, since, at this stage, the ACA decomposition cannot fully represent all the eigenvectors of $A$, so the error still depends on these eigenvectors. Hence, we focus on the evolution beyond this point.

Consider the alternative, equivalent representation of the ACA decomposition [10]

$$
A(:, \mathbf{j}) A(\mathbf{i}, \mathbf{j})^{-1} A(\mathbf{i},:) \approx A
$$

where $\mathbf{i}$ and $\mathbf{j}$ are vectors of length $k$ containing the indices of the ACA selected rows and columns, $I_{k}$ and $J_{k}$, respectively. In [10], this representation is shown to be equivalent to a two-sided Lagrange (polynomial) interpolation of the elements. Suppose that the rows of $A$ represent sources at points $\mathbf{x}$ in domain $D_{X}$, and the columns represent fields at points $\mathbf{y}$ in domain $D_{Y}$. Then, the approximate value of the matrix element $A(p, q)$ representing the field at $\mathbf{y}_{q}$ due to a source at $\mathbf{x}_{p}$ is found by polynomial interpolation through the values at $\mathbf{y}_{\mathbf{j}}$ and $\mathbf{x}_{\mathbf{i}}$. Polynomial interpolation yields the exact value at the interpolation points, while in between, the error may oscillate depending on the polynomial order. However, as the density of interpolation points increases, the error will be progressively locally linearizable and become proportional to the distance from the nearest interpolation point. The ACA selects the interpolation points on the basis of the largest current error at each iteration. Once we are beyond $k=K$, the points are therefore chosen as far as possible from any neighbor, leading to a progressively more uniform distribution over the two domains.

In [11], it is proven that given a set of points, uniformly distributed over an $N$-dimensional domain, the probability for any location within that domain to find any of these points within a Euclidean distance $d$ is exponentially distributed. Since, as argued earlier, we have, beyond $k=K$, a linear relationship between the error and the distance, and the interpolation error on both sides of the ACA approximation should be exponentially distributed too. Hence, $\mathbf{u}_{i}$ and $\mathbf{v}_{i}$ in (4) are exponentially distributed, for large enough $i$. This does not immediately reveal the distribution of $E_{k}$ itself, but we can invoke the same reasoning that leads to the conventional ACA convergence criterion; once we are in the range of exponential decrease of $\left|E_{k}\right|, \mathbf{u}_{k} \mathbf{v}_{k}^{T}$ is the dominant contribution, and we assume that the distribution of $E_{k}$ is close to that of $\mathbf{u}_{k} \mathbf{v}_{k}^{T}$.

Before proceeding to put this model to the test, we need to address two additional considerations. First, we are ultimately interested in the distribution of the squared norms of the elements of $E_{k}$ rather than the elements of $E_{k}$ themselves, and second, we are dealing with complex matrices, with the real and imaginary parts identically and independently distributed (i.i.d.), following the exponential distribution. If we denote $\mathrm{U}_{\mathrm{R}}, \mathrm{U}_{\mathrm{I}} \sim \exp \left(\lambda_{u}\right)$ and $\mathrm{V}_{\mathrm{R}}, \mathrm{V}_{\mathrm{I}} \sim \exp \left(\lambda_{v}\right)$ (where $\sim$ means "has the distribution") as the random variables representing the real and imaginary parts of the elements of $\mathbf{u}_{k}$ and $\mathbf{v}_{k}$, respectively, then $\left|E_{k}\right|^{2}$ is distributed as $\mathrm{W}$, with

$$
\mathrm{W} \sim\left(\mathrm{U}_{\mathrm{R}}^{2}+\mathrm{U}_{\mathrm{I}}^{2}\right)\left(\mathrm{V}_{\mathrm{R}}^{2}+\mathrm{V}_{\mathrm{I}}^{2}\right)
$$

or equivalently

$$
\mathrm{W} \sim\left(\mathrm{U}_{\mathrm{R}} \mathrm{V}_{\mathrm{R}}\right)^{2}+\left(\mathrm{U}_{\mathrm{R}} \mathrm{V}_{\mathrm{I}}\right)^{2}+\left(\mathrm{U}_{\mathrm{I}} \mathrm{V}_{\mathrm{R}}\right)^{2}+\left(\mathrm{U}_{\mathrm{I}} \mathrm{V}_{\mathrm{I}}\right)^{2} .
$$

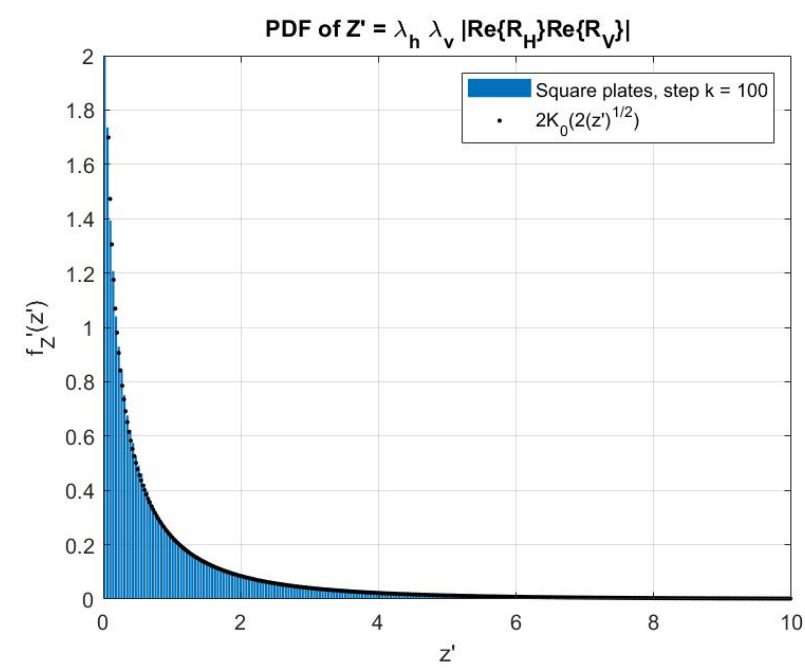

Fig. 7. Measured and theoretical probability density function of $Z^{\prime}$ for the case of Fig. 5.

The distribution of $\mathrm{W}$ cannot be expressed in closed form, as far as we know. However, knowing that the expectation of the exponential distribution equals $\mathrm{E}(\exp (\lambda))=1 / \lambda$ and using some elementary probabilistic identities for the expectation of sums and products of independent random variables, we find from (6): $\mathrm{E}(\mathrm{W})=16 /\left(\lambda_{u} \lambda_{v}\right)^{2}$. To know more about W, we turn to the representation in (7). The four terms on the right-hand side are squares of products of two exponential distributions, each term involving the two scale parameters $\lambda_{u}$ and $\lambda_{v}$. The product of two exponential distributions $\exp \left(\lambda_{u}\right)$ and $\exp \left(\lambda_{v}\right)$ yields the K-distribution [12]

$$
\mathrm{Z} \sim f_{Z}(x)=2 \lambda_{u} \lambda_{v} K_{0}\left(2 \sqrt{\lambda_{u} \lambda_{v} x}\right)
$$

where $\mathrm{Z}$ is any of the four terms in (7) and $K_{0}$ denotes the modified Bessel function of the second kind and order zero. Observe that $Z^{\prime}=\lambda_{u} \lambda_{v} Z$ yields a parameter-free distribution: $Z^{\prime} \sim 2 K_{0}(2 \sqrt{x})$. To demonstrate the validity of this model, even in the case of an irregular mesh, Fig. 7 shows the distribution of $\left|\operatorname{Re}\left\{\mathbf{u}_{k}\right\} \operatorname{Re}\left\{\mathbf{v}^{\mathbf{T}}{ }_{k}\right\}\right|$, normalized to the mean for step $k=100$ of an execution of the ACA for the square plate problem of Fig. 5, together with the theoretical PDF of $Z^{\prime}$.

Since $\mathrm{W} \sim \sum_{i=1}^{4} \mathrm{Z}^{2}=\left(\lambda_{u} \lambda_{v}\right)^{2} \sum_{i=1}^{4} \mathrm{Z}^{\prime 2}$, we note that $\mathrm{W}^{\prime}=\mathrm{W} / \mathrm{E}(\mathrm{W})$ is also parameter-free. In other words, the distribution of $\left|E_{k}\right|^{2}$ normalized by its mean converges, for large enough iteration step $k$, to a unique, parameter-free distribution.

We can now also calculate the coefficient of variation $C V$, defined as $\sigma / \mu$ (the standard deviation over the mean), of $\mathrm{W}^{\prime}$ (and $\mathrm{W}$ ). This is difficult using (7), because the terms are not independent, but we can use (6). The square of an $\exp (\lambda)$ distribution is easily shown to be a Weibull $\left(1 / 2,1 / \lambda^{2}\right)$ distribution [13], which has $C V_{\mathrm{Wei}(1 / 2)} \approx 2.23$. From (6), $\mathrm{W}$ is the product of two sums of such distributions, all of them i.i.d. Using the following relation for i.i.d. random variables $\mathrm{X}$ and $\mathrm{Y}$ :

$$
C V_{\mathrm{XY}}=\sqrt{C V_{\mathrm{X}}^{2}+C V_{\mathrm{Y}}^{2}+C V_{\mathrm{X}}^{2} C V_{\mathrm{Y}}^{2}}
$$

we find $C V_{\left|E_{k}\right|^{2}} \approx C V_{\mathrm{W}} \approx 3.35$. 


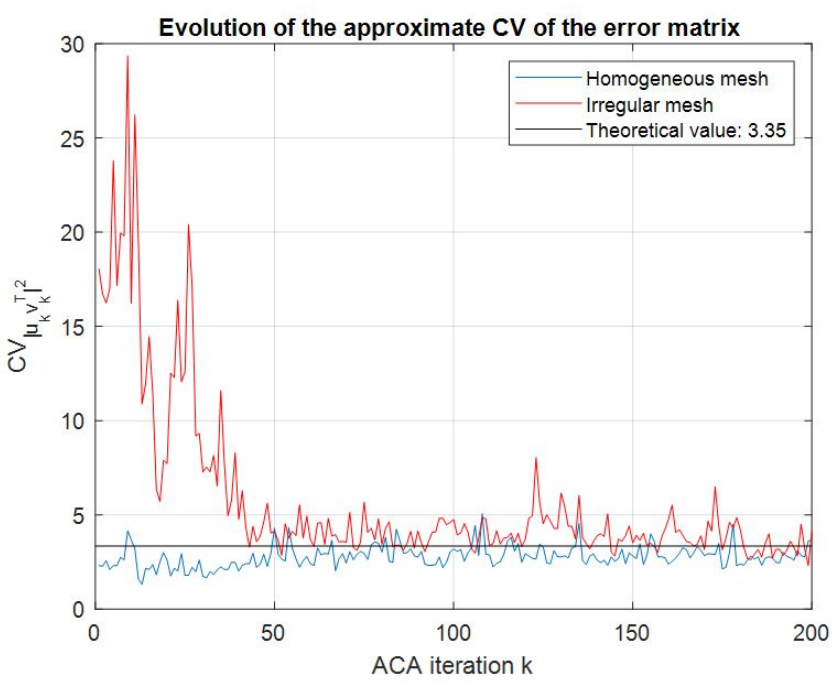

Fig. 8. Approximate coefficient of variation of the distribution of the squared norms of the error matrix elements as a function of the ACA iteration step.

Of course, we cannot compute the true $C V$ of $\left|E_{k}\right|^{2}$ itself at every step, but since as shown in [1] and [2], $u_{k} v_{k}^{T} \approx E_{k}$ and $C V_{\left|u_{k} v_{k}^{T}\right|^{2}}$ can be used as an approximation of $C V_{\left|E_{k}\right|^{2}}$. Computing $C V_{\left|u_{k} v_{k}^{T}\right|^{2}}$ is fast; first, we find $C V_{\left|u_{k}\right|^{2}}$ and $C V_{\left|v_{k}\right|^{2}}$ by computing the mean and variance of the respective vectors. Then, we use (9) to compute the final result.

Now, we can predict a characteristic value of the distribution of $\left|E_{k}\right|^{2}$ and can test the model on the problem of the interaction between two square plates presented in Section IV. In Fig. 8, we see the $C V$ of the elements of $\left|\mathbf{u}_{k} \mathbf{v}_{k}^{T}\right|^{2}$ for each step $k$, both for the case of the homogeneous mesh and the highly irregular mesh. We see that with the homogeneous mesh, initially, the $C V$ is, on average, somewhat below the theoretical value. Eventually, the $C V$ does converge toward the theoretical value. In the case of the irregular mesh, we see that indeed, the $C V$ varies wildly initially, assuming very high values. However, after about 40 steps, it also converges toward the theoretical value.

Just like in the case of the conventional convergence criterion, the accuracy of the approximation $C V_{\left|u_{k} v_{k}^{T}\right|^{2}} \approx C V_{\left|E_{k}\right|^{2}}$ is impossible to quantify. What we can do is to compare the approximation with the true $C V$ of the population distribution of $\left|E_{k}\right|^{2}$. The true $C V$ is shown in Fig. 9. We observe that qualitatively, there is a good correspondence, but there is a clear discrepancy; the true $C V$ is always somewhat lower. In fact, the true distribution is a weighted sum of the distributions of $\mathbf{u}_{i} \mathbf{v}_{i}^{T}$ of the last few iterations. Assuming that these are all the same (equal to $\mathrm{W}$ ), the distribution will be that of a linear combination of i.i.d. distributions with decreasing weights. This by elementary probability theory will always yield a smaller $C V$ than the terms individually.

\section{Enhanced New ACA Convergence Criterion}

With the convergence criterion of Section III, the average number of steps needed for convergence to $\tau=10^{-3}$ is 37,7 for the two squares with homogeneous mesh and 37.6 for

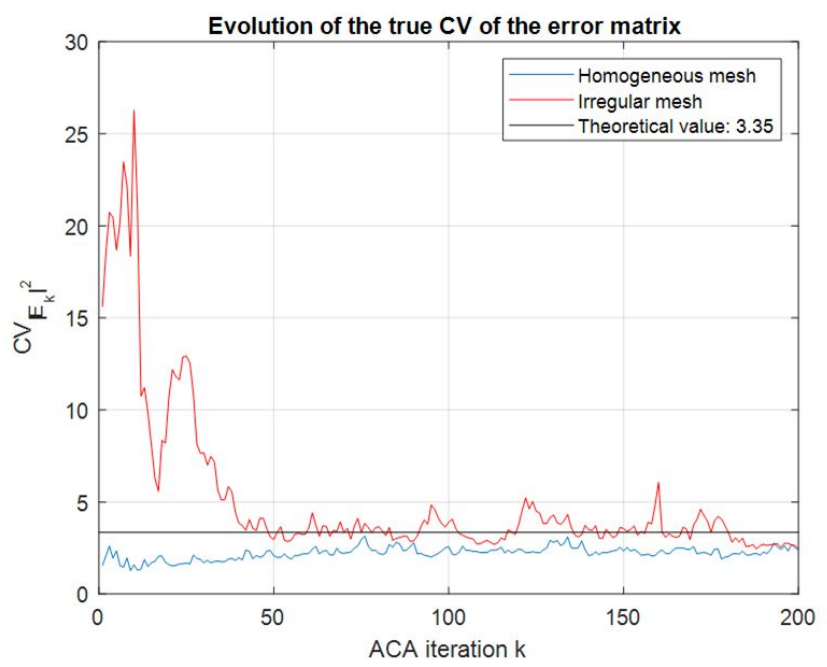

Fig. 9. True coefficient of variation of the distribution of the squared norms of the error matrix elements as a function of the ACA iteration step.

the irregular mesh, almost the same, as expected since the underlying physical problem is the same in both cases. Notice that for the irregular mesh, the number of steps before the $C V$ converges to the critical value of 3.35 is larger than the average number of steps for convergence of the ACA. This means that often, the sample that detects convergence is taken from a distribution with a very high $C V$, which explains the large spread in the true relative error in Fig. 6 with $M=100$.

We might be tempted to use the measured $C V$ at step $k$ directly to compute a shifted threshold $\tau^{\prime}$ in order to ensure that the true mean lies below the real $\tau$ with a chosen probability, for example using Tchebichef's inequality [14]. The main problem with this approach is precisely the fact that it is based only on the first two moments of the distribution: $\mu$ and $\sigma^{2}$. The first two moments do not reveal anything about the asymmetry of the distribution. Yet, the typical "difficult" distribution is highly asymmetric, many small elements and a few very large ones. This leads to a long right tail in the distribution and a high $C V$. In reality, we are more interested in the left tail: the risk of an underestimation of the mean (and hence a premature convergence) is determined here.

In fact, we used the $C V$ to test the theory developed in Section IV, but, under the assumption that the theory is correct, we know much more than the first two moments. Although we do not have a closed expression for the distribution, we do know that after sufficient steps, it is always the same, independent of the underlying problem or the discretization. We propose to use the measured approximate value of the $C V$ as a test for how closely the error distribution probability density at step $k$ corresponds to $f_{\mathrm{W}}$. Once it is close enough, we will obtain, for a chosen sample size, a fixed distribution of the true relative error. Hence, we supplement the convergence criterion based on the sample mean with a second condition, namely

$$
C V_{\left|u_{k} v_{k}^{T}\right|^{2}}<C V_{\text {crit }}
$$




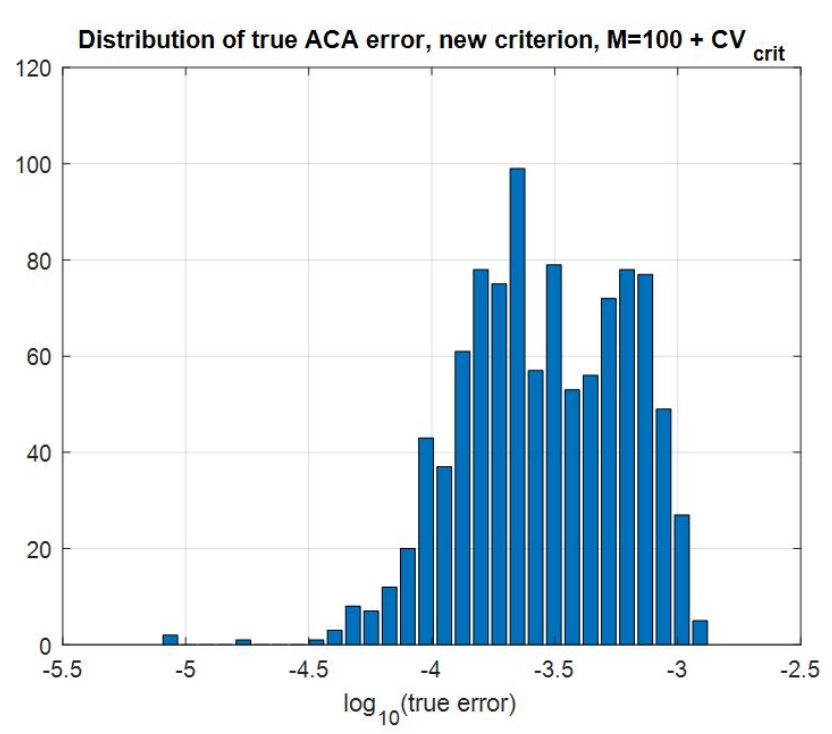

Fig. 10. Histogram of the true relative error for the same problem as in Fig. 5, with the proposed new criterion, $M=100$, and imposing $C V_{\left|u_{k} v_{k}^{T}\right|^{2}}<4$, 1.000 runs.

The exact choice of $C V_{\text {crit }}$ should not be highly consequential, as long as the excessive peaks observed in Fig. 8 are rejected. We suggest $C V_{\text {crit }}=4$, just above the theoretical value of 3.35 .

A consequence of this approach is of course that for "difficult" matrix blocks, such as that of Fig. 5, we risk ending up taking far more ACA steps than necessary and obtaining a precision far beyond the required value. However, most ordinary blocks will always comply with (10). Typically, large simulations require compressing many blocks, most of which will be "ordinary," so the overall computational overhead due to (10) will be very limited. In essence, (10) allows the algorithm to "cheaply" detect whether a block is "difficult," in which case it is treated with extraordinary precaution. Fig. 10 shows the effect of imposing (10) on the result for the two plates with irregular mesh. Effectively, the convergence takes much longer than before: 47.2 steps on average. However, the desired accuracy is clearly obtained. Typically, the proportion of such difficult cases will be so small that dedicating some extra effort to them will have a negligible influence on the overall computation time. Meanwhile, risking an inaccurate ACA approximation of just one of them may seriously compromise the overall accuracy.

We conclude this section with a listing of the ACA algorithm following [2], with the new convergence criterion incorporated (the bold-faced lines).

\section{Initialization :}

1) Store $M$ random row/column indices in $\mathbf{r}$ and $\mathbf{c}$.

2) $\mathrm{e}_{0}(i)=A\left(\mathrm{r}_{i}, \mathrm{c}_{i}\right), \quad i=1 \cdots M$.

3) Initialize the first row index $I_{1}=1$.

4) Initialize error matrix: $\widetilde{\mathbf{v}}_{1}=A\left(I_{1},:\right)$.

5) Find $J_{1}=\max _{j}\left(\left|\widetilde{\mathbf{v}}_{1}\right|\right)$.

6) $\mathbf{v}_{1}=\widetilde{\mathbf{v}}_{1} / \widetilde{\mathbf{v}}_{1}\left(J_{1}\right)$.

7) $\mathbf{u}_{1}=A\left(:, J_{1}\right)$.

8) $\left\|\widetilde{Z}^{(1)}\right\|^{2}=\left(\left|\mathbf{u}_{k}\right|\left|\mathbf{v}_{k}\right|\right)^{2}$.

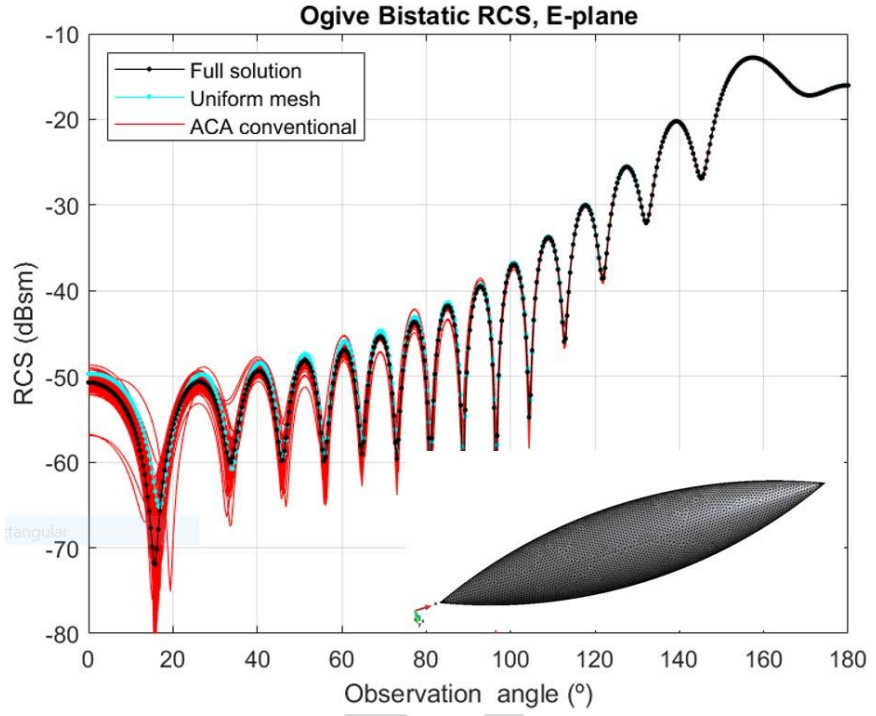

Fig. 11. E-plane Bistatic RCS of a metallic ogive, incidence along the ogive axis. Black dots: solution by inversion of the full impedance matrix. Red curves: 250 different ACA computations with the conventional convergence criterion. Blue dots: full solution using a mesh without refinement at the tips.

9) Find $I_{2}=\max _{i}\left(\left|\mathbf{u}_{1}\right|\right), i \neq I_{1}$.

$k^{\text {th }}$ Iteration:

1) $\widetilde{\mathbf{v}}_{k}=A\left(I_{k},:\right)-\sum_{l=1}^{k-1}\left(\mathbf{u}_{l}\right)_{I_{k}} \mathbf{v}_{l}$

2) Find $J_{k}=\max _{j}\left(\left|\widetilde{\mathbf{v}}_{k}\right|\right), j \neq J_{1} \cdots J_{k-1}$.

3) $\mathbf{v}_{k}=\widetilde{\mathbf{v}}_{k} / \widetilde{\mathbf{v}}_{k}\left(J_{k}\right)$.

4) $\mathbf{u}_{k}=A\left(:, J_{k}\right)-\sum_{l=1}^{k-1}\left(\mathbf{v}_{l}\right)_{J_{k}} \mathbf{u}_{l}$.

5) $\left\|\widetilde{Z}^{(k)}\right\|^{2}=\left\|\widetilde{Z}^{(k-1)}\right\|^{2}+2 \operatorname{Re}\left\{\sum_{j=1}^{k-1}\left(\mathbf{u}_{j}^{T} \mathbf{u}_{k}\right) \cdot\left(\mathbf{v}_{j}^{T} \mathbf{v}_{k}\right)\right\}+$ $+\left(\left|\mathbf{u}_{k}\right|\left|\mathbf{v}_{k}\right|\right)^{2}$

6) $\mathrm{e}_{k}(i)=\mathrm{e}_{k-1}(i)-\mathbf{u}_{k}\left(\mathrm{r}_{i}\right) \mathbf{v}_{k}\left(\mathbf{c}_{i}\right), i=1 \cdots M$

7) Compute the $C V$ of $\left|\mathbf{u}_{k}\right|^{2}$ and $\left|\mathbf{v}_{k}\right|^{2}$.

8) $C V_{e}=\sqrt{C V_{u}^{2}+C V_{v}^{2}+C V_{u}^{2} C V_{v}^{2}}$

9) If mean $\left(\left|\mathrm{e}_{k}^{2}\right|\right)<\frac{\tau}{n m}\left\|\tilde{Z}^{(k)}\right\|^{2^{u}}$ AND $C V_{e}<4$ exit.

10) Find $I_{k+1}=\max _{i}\left(\left|\mathbf{u}_{k}\right|\right), i \neq I_{1} \cdots I_{k}$.

Note : Line 5 of the iterations is erroneous in the original paper [2]. It is corrected here following [15].

\section{PRactical Example}

Providing a large-scale example of the merits of the new criterion is difficult because we need to execute the simulation many times with random initial row indices. Furthermore, we must be able to solve the full system directly to provide a reference solution. We, therefore, present a relatively small problem. The target is the metallic ogive defined in [16] (see the inset in Fig. 11), at $9 \mathrm{GHz}$. The ogive surface is discretized with an average mesh size of $0.003 \mathrm{~m}$, but with a refinement of the mesh near the tips where the mesh size is approximately ten times smaller. This leads to $24.804 \mathrm{RWG}$ basis functions (the uniform mesh, without the refinement has 23.472 basis functions). We decompose the ogive surface into 100 subdomains with an approximately equal number of basis functions each. This leads to very small blocks (about $250 \times 250$ ), which may not be the most efficient choice, but it mimics a large-scale problem that typically has several 


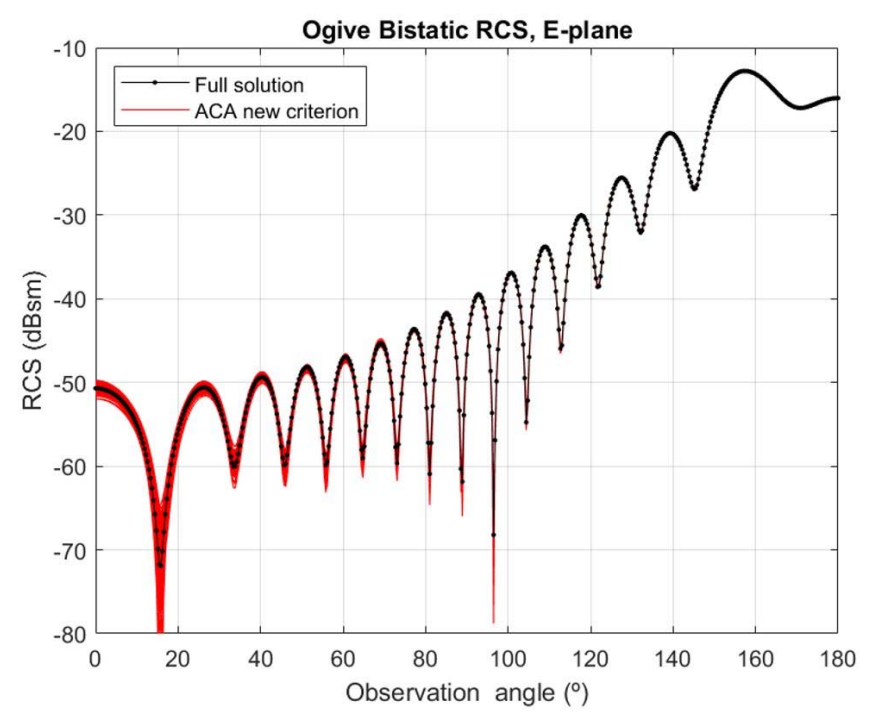

Fig. 12. E-plane Bistatic RCS of a metallic ogive, incidence along the ogive axis. Black dots: solution by inversion of the full impedance matrix. Red curves: 250 different ACA computations with the proposed new convergence criterion.

hundreds or thousands of subdomains. Since this is not a particularly large or badly conditioned problem, we use an ACA convergence threshold of $\tau=0.01$. We solve for the surface current density using a GMRES iterative solver with a preconditioner according to [17]. Then, we calculate the bistatic RCS for an angle of incidence along the positive $x$-axis, parallel to the ogive axis. Figs. 11 and 12 show the E-plane RCS from 250 calculations using the ACA without and with the new convergence criterion, respectively, compared with the full solution. Also shown is the full solution for the uniform mesh, to show that the mesh refinement does indeed affect the result.

It is clear that the new criterion leads to considerably more accurate results. In fact, the median relative error in the surface current vector equals, for the new criterion, $4 \%$, with a maximum of $10 \%$. For the conventional criterion, the median is $13 \%$ and the maximum error is $800 \%$. The difference in medians indicates that the new criterium is consistently a factor 3 more accurate. However, more importantly, it avoids the occasional outliers of the conventional ACA. Interestingly, even the enormous maximum error of the conventional ACA still yields a reasonable RCS. Looking at the detail of the surface current solution reveals that this large error is localized very near one of the tips. Since the RCS computation integrates over the entire surface, a very large but very localized error may well have a limited effect on the RCS. For problems with larger, badly conditioned impedance matrices, we expect the error to spread throughout the solution, invalidating any far-field results.

Regarding the size of the compressed matrix, with the conventional ACA, it equals on average $689 \mathrm{MB}$ (double precision, exploiting symmetry to store only half the matrix), of which $384 \mathrm{MB}$ are the self-block and touching block that are not compressed. Therefore, the compressed matrices take up on average $305 \mathrm{MB}$, with very little variation. Using the new criterion, the average size of the compressed matrices equals 299 MB. Surprisingly, the new criterion yields slightly more compression. The average matrix construction time is $36.4 \mathrm{~s}$ with the conventional criterion and $37.5 \mathrm{~s}$ with the new criterion, about $3 \%$ more. Profiling the code, we find that this is entirely due to the computation of 100 sample elements (line 2 of the initialization phase in the algorithm). The problem is that it takes much more time to compute individual matrix elements than to fill entire rows or columns at once. This is due to the fact that our MoM discretization for a triangular mesh is based on Rao, Wilton, and Glisson basis and testing functions [8]. Computing $M$ random elements of the impedance matrix needs integration on $4 M$ triangles, while computing a full row or column of $M$ elements needs $4 M / 3$. In addition, computing full rows or columns makes better use of the computer CPU cache memory.

\section{CONCLUSION}

A new ACA convergence criterion has been proposed that avoids the high inaccuracy, of more than a factor 10, sometimes produced by the unpredictable errors caused by the conventional ACA convergence criterion. The new criterion uses a global sampling of the error matrix. The sampling error inherent in this approach can be controlled by exploiting the fact that once the matrix to be compressed is fully represented by its low-rank ACA approximation, the error matrix elements are distributed according to a unique probability density function such that the sampling error only depends on the sample size. A complementary criterion is proposed to check whether this distribution has been established. Although in this article the proposed method has only been applied to the ACA, it may be useful for estimating the residual error in any algorithm that approximates low-rank matrices by interpolation of a reduced set of its elements.

\section{REFERENCES}

[1] M. Bebendorf, "Approximation of boundary element matrices," Numerische Math., vol. 86, no. 4, pp. 565-589, Oct. 2000.

[2] K. Zhao, M. N. Vouvakis, and J.-F. Lee, "The adaptive cross approximation algorithm for accelerated method of moments computations of EMC problems," IEEE Trans. Electromagn. Compat., vol. 47, no. 4, pp. 763-773, Nov. 2005

[3] J. Laviada, R. Mittra, M. R. Pino, and F. Las-Heras, "On the convergence of the ACA," Microw. Opt. Technol. Lett, vol. 51, no. 10, pp. 2458-2460, Oct. 2009

[4] A. Heldring, E. Ubeda, and J. M. Rius, "On the convergence of the ACA algorithm for radiation and scattering problems," IEEE Trans. Antennas Propag., vol. 62, no. 7, pp. 3806-3809, Jul. 2014.

[5] N. Halko, P. G. Martinsson, and J. A. Tropp, "Finding structure with randomness: Probabilistic algorithms for constructing approximate matrix decompositions," SIAM Rev., vol. 53, no. 2, pp. 217-288, Jan. 2011.

[6] M. W. Mahoney and P. Drineas, "CUR matrix decompositions for improved data analysis," Proc. Nat. Acad. Sci. USA, vol. 106, no. 3, pp. 697-702, Jan. 2009.

[7] A. A. Kashirin, S. I. Smagin, and M. Y. Taltykina, "Mosaic-skeleton method as applied to the numerical solution of three-dimensional Dirichlet problems for the Helmholtz equation in integral form," Comput. Math. Math. Phys., vol. 56, no. 4, pp. 612-625, Apr. 2016.

[8] S. Rao, D. Wilton, and A. Glisson, "Electromagnetic scattering by surfaces of arbitrary shape," IEEE Trans. Antennas Propag., vol. AP-30, no. 3, pp. 409-418, May 1982.

[9] Y. Saad and M. H. Schultz, "GMRES: A generalized minimal residual algorithm for solving nonsymmetric linear systems," SIAM J. Sci. Stat. Comput., vol. 7, no. 3, pp. 856-869, Jul. 1986. 
[10] M. Bebendorf and R. Venn, "Constructing nested bases approximations from the entries of non-local operators," Numerische Math., vol. 121, no. 4, pp. 609-635, Aug. 2012.

111] M. Jirina and M. Jirina, "Features of neighbors spaces," SOFSEM 2004: Theory and Practice of Computer Science (Lecture Notes in Computer Science), vol. 2932. 2004, pp. 241-248.

[12] N. J. Redding, "Estimating the parameters of the $\mathrm{k}$ distribution in the intensity domain," DSTO Electron. Surveill. Lab., South Australia, Tech. Rep. DSTO-TR-0839, Jul. 1999.

13] N. L. Johnson, S. Kotz, Samuel, and N. Balakrishnan, Continuous Univariate Distributions (Wiley Series in Probability and Mathematical Statistics). vol. 1. 1994

[14] P. Tchebichef, "Des valeurs moyennes," J. de Mathématiques Pures et Appliquées, vol. 2, no. 12, pp. 177-184, 1867.

[15] X. Chen, C. Gu, J. Ding, Z. Li, and Z. Niu, "Multilevel fast adaptive cross-approximation algorithm with characteristic basis functions," IEEE Trans. Antennas Propag., vol. 63, no. 9, pp. 3994-4002, Sep. 2015.

[16] A. C. Woo, H. T. G. Wang, M. J. Schuh, and M. L. Sanders "Benchmark radar targets for the validation of computational electromagnetics programs," IEEE Antennas Propag. Mag., vol. 35, no. 1, pp. 84-89, Feb. 1993

17] A. Heldring, E. Ubeda, and J. M. Rius, "The multiscale compressed block decomposition as a preconditioner for method of moments computations," in Proc. 7th Eur. Conf. Antennas Propag. (EuCAP), Gothenborg, Sweden, Apr. 2013, pp. 8-12.

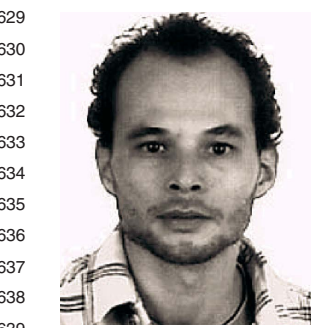

Alexander Heldring was born in Amsterdam, The Netherlands, in 1966. He received the M.S. degree in applied physics and the Ph.D. degree in electrical engineering from the Delft University of Technology, Delft, The Netherlands, in 1993 and 2002, respectively.

$\mathrm{He}$ is currently an Associate Professor with the Telecommunications Department, Universitat Politècnica de Catalunya (UPC), Barcelona, Spain. He has authored over 30 articles in international journals and 100 in international conference proceedings. His special research interest includes fast integral equation methods for electromagnetic problems.

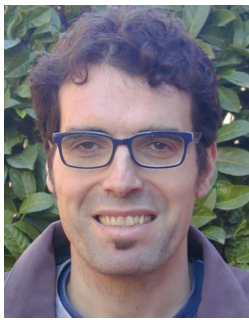

Eduard Ubeda (Member, IEEE) was born in Barcelona, Spain, in 1971. He received the Telecommunication Engineer and Doctor Ingeniero degrees from the Universitat Politècnica de Catalunya (UPC), Barcelona, in 1995 and 2001, respectively.

In 1996, he was with the Joint Research Center from the European Commission, Ispra, Italy. From 1997 to 2000, he was a Research Assistant with the Electromagnetic and Photonic Engineering Group, UPC. From 2001 to 2002, he was a Visiting Scholar with the Electromagnetic Communication Laboratory, Electrical Engineering Department, Pennsylvania State University (PSU), University Park, PA, USA. Since 2003, he has been with the Universitat Politècnica de Catalunya (UPC). He has authored over 30 articles in international journals and 100 in international conference proceedings. His main research interests are numerical computation of scattering and radiation using integral-equation methods and advanced discretization techniques.

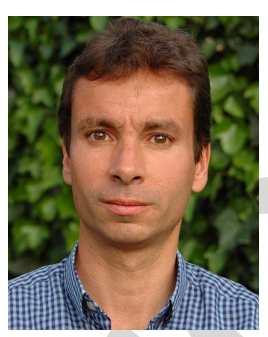

Juan M. Rius (Senior Member, IEEE) was born in Barcelona, Spain, in 1963. He received the "Ingeniero de Telecomunicación" and "Doctor Ingeniero" degrees from the Universitat Politècnica de Catalunya (UPC), Barcelona, in 1987 and 1991, respectively.

In 1985 , he joined the Electromagnetic and Photonic Engineering Group, UPC, now the CommSensLab, where he currently holds a position of "Catedrático" (equivalent to Full Professor). From 1985 to 1988 , he developed a new inverse scattering algorithm for microwave tomography in cylindrical geometry systems. Since 1989, he has been involved in the research for new and efficient methods for numerical computation of electromagnetic scattering and radiation. He is the Developer of the graphical electromagnetic computation (GRECO) approach for high-frequency RCS computation, the Integral Equation formulation of the Measured Equation of Invariance (IE-MEI), and the multilevel matrix decomposition algorithm (MLMDA) in 3-D. He has held positions of "Visiting Professor" at EPFL, Lausanne, Switzerland, from May 1, 1996 to October 31, 1996; a "Visiting Fellow" at the City University of Hong Kong, Hong Kong, from January 3, 1997 to February 4, 1997; the "CLUSTER Chair" at EPFL from December 1, 1997 to January 31, 1998; and a "Visiting Professor" at EPFL from April 1, 2001 to June 30, 2001. He has more than 70 articles published or accepted in refereed international journals (40 in IEEE Transactions) and more than 200 in international conference proceedings. His current interests are the numerical simulation of electrically large antennas and scatterers. 